\title{
Mikroplastik: die Geister, die wir riefen
}

\author{
Darena Schymanski ${ }^{1}$
}

Published online: 16 February 2019

(C) Bundesamt für Verbraucherschutz und Lebensmittelsicherheit (BVL) 2019

Haben Sie schon einmal Mikroplastik gegoogelt? Was stellen Sie fest? Vermutlich: Mikroplastik ist gesundheitsschädlich und überall. Sogar im Stuhl! Obwohl bislang keine negativen Auswirkungen auf den Menschen bekannt sind, steht Mikroplastik laut dem letzten Verbrauchermonitor des Bundesinstituts für Risikobewertung auf Platz zwei der Gesundheitsthemen, die Verbraucher beunruhigen.

Werde ich als Wissenschaftlerin [die zu diesem Thema forscht, Anm. d. Red.] von Freunden oder Bekannten gefragt „Ist Mikroplastik wirklich so schlimm?“ antworte ich: „So einfach ist das nicht.“ Denn wenn wir uns die bisher bekannten Fakten näher ansehen, müssen wir feststellen, dass diese allein nicht das Problem sind.

\section{Plastik ist persistent}

Beunruhigend ist: Auch wenn inzwischen einige wenige Organismen gefunden wurden, die Polymere zersetzen können, wie zum Beispiel das Bakterium Ideonella sakaiensis 201-F6, ${ }^{1}$ gelten die meisten Plastiksorten als nicht biologisch abbaubar. Seit Beginn der Kunststoffherstellung in den 1950er-Jahren wurden schätzungsweise 8,3 Milliarden Tonnen produziert, ${ }^{2}$ die Hälfte davon in den letzten 13 Jahren. Wenn sich die Primärproduktion nicht vermindert und der Umgang mit dem Abfall sich nicht ändert, prognostizieren Geyer und Mitarbeiter 12 Milliarden Tonnen Plastikmüll im Jahr 2050. Dass uns der bisher weltweit in die Umwelt gelitterte ${ }^{3}$ und auf Deponien gelagerte Anteil, nämlich ca. $80 \%$ der 6,5 Millionen Tonnen Plastikmüll, die bis 2015 erzeugt wurden ${ }^{2}$, noch nicht überflutet, liegt auch daran, dass sich Plastikmüll unter bestimmten Bedingungen allmählich zersetzt. Alle als Makroplastik bezeichneten, größeren Kunststoffstücke, die

Darena Schymanski

darena.schymanski@cvua-mel.de

1 Chemisches Und Veterinäruntersuchungsamt MünsterlandEmscher-Lippe (CVUA-MEL), Münster, Germany als Müll im Meer treiben oder am Wegesrand liegen, werden mit der Zeit brüchiger und kleiner, bis sie mikrometerklein und für das bloße Auge unsichtbar sind.

Bei genauerem Hinsehen - mit geeigneten, mikrospektroskopischen Methoden - stellen wir dann fest, dass sich dieses sekundäre Mikroplastik überall verteilt. So könnte eine Plastiktüte durch den Einfluss von Wellenbewegung und UV-Strahlung zu vielen Millionen Mikroplastik-Partikeln fragmentieren und diese sich durch Winde und Strömungen in jede Ecke der Welt verteilen. In allen Weltmeeren $^{4}$ und Oberflächengewässern, ${ }^{5}$ Tiefseesedimenten $^{6}$ und Küstengebieten ${ }^{7}$ und sogar im Polareis ${ }^{8}$ wurden diverse Sorten und Größen von Kunststoffteilchen nachgewiesen. Auch eine Verteilung über die Luft ist möglich. ${ }^{9}$

\footnotetext{
1 Yoshida S, Hiraga K, Takehana T, Taniguchi I, Yamaji H, Maeda Y, Toyohara K, Miyamoto K, Kimura Y, Oda K (2016) A bacterium that degrades and assimilates poly(ethylene terephthalate). Science 351:1196-1199.

2 Geyer R, Jambeck JR, Law KL (2017) Production, use, and fate of all plastics ever made. Sci Adv 3:e1700782.

${ }^{3}$ Littering: Verschmutzung durch Wegwerfen oder Liegenlassen von Müll.

${ }^{4}$ Cózar A, Echevarría F, González-Gordillo JI, Irigoien X, Ubeda B, Hernández-León S, Palma AT, Navarro S, García-de-Lomas J, Ruiz A, Fernandez-de-Puelles ML, Duarte CM (2014) Plastic debris in the open ocean. Proc Natl Acad Sci USA 111:10239-10244.

${ }^{5}$ Li J, Liu H, Paul Chen J (2018) Microplastics in freshwater systems: A review on occurrence, environmental effects, and methods for microplastics detection. Water Res 137:362-374.

${ }^{6}$ Woodall LC, Sanchez-Vidal A, Canals M, Paterson GLJ, Coppock R, Sleight V, Calafat A, Rogers AD, Narayanaswamy, BE, Thompson RC (2014) The deep sea is a major sink for microplastic debris. R Soc Open Sci 1:140317.

7 Browne MA, Crump P, Niven SJ, Teuten E, Tonkin A, Galloway T, Thompson R (2011) Accumulation of microplastic on shorelines worldwide: sources and sinks. Environ Sci Technol 45:9175-9179.

${ }^{8}$ Obbard RW, Sadri S, Wong YQ, Khitun AA, Baker I, Thompson RC (2014) Global warming releases microplastic legacy frozen in Arctic Sea ice. Earth's Future 2:315-320.

${ }^{9}$ Dris R, Gasperi J, Saad M, Mirande C, Tassin B (2016) Synthetic fibers in atmospheric fallout: a source of microplastics in the environment? Mar Pollut Bull 104:290-293.
} 
Das ist jedoch nur ein Eintragsweg von sekundärem Mikroplastik. Viel gravierender ist laut einer Hochrechnung des Fraunhofer Instituts ${ }^{10}$ Reifenabrieb, erst dann folgt die Abfallentsorgung.

Primäres Mikroplastik sind Kunststoffpartikel, die für bestimmte Verwendungszwecke in Mikrometergröße hergestellt werden. Diese können beispielsweise aus kosmetischen Mitteln indirekt über den Abwasserpfad und Klärschlamm in die Umwelt gelangen. Der Anteil des primären Mikroplastiks an der Gesamtbelastung wird als jedoch verhältnismäßig gering eingeschätzt.

Was passiert nun mit den Mikroplastik-Partikeln, die im Meer treiben, in der Luft schweben oder zu Boden sinken? Sie können Schadstoffe aus der Umgebung anlagern und diese wieder im Organismus abgeben. ${ }^{11}$ Sie können von marinen Organismen aufgenommen werden. ${ }^{12}$ Auch eine mögliche Übertragung zwischen den Trophieebenen ${ }^{13}$ wurde in der Presse beschrieben, kürzlich sogar ,,von der Mücke in Vögel und Fledermäuse“. ${ }^{14}$ Das klingt dramatisch und birgt eine potenzielle Gefahr, die keineswegs unterschätzt werden darf. Alle Studien jedoch, die negative Effekte, wie beispielsweise Entzündungsreaktionen bei Muscheln oder Verhaltensänderungen bei Fischen, nachgewiesen haben, verwendeten hohe Mikroplastik-Konzentrationen, wie sie bisher in der Natur nicht nachgewiesen wurden. In der Elbe kommen auf 10 Mikroplastik-Partikel rund 1.000.000 natürliche Partikel. ${ }^{15}$ In oben erwähnter Studie $^{16}$ wurden Mückenlarven in Lösungen mit 80.000 Mikroplastik-Partikeln je Milliliter aufgezogen. In den geschlüpften Mücken wurden nur ca. 40 davon gezählt. Trotzdem hat die Presse das Insektensterben und den

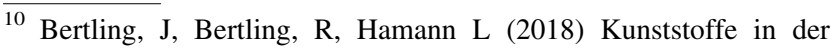
Umwelt: Mikro- und Makroplastik. Ursachen, Mengen, Umweltschicksale, Wirkungen, Lösungsansätze, Empfehlungen. Kurzfassung der Konsortialstudie, Fraunhofer-Institut für Umwelt-, Sicherheits- und Energietechnik UMSICHT, Oberhausen, Juni 2018.

11 Teuten EL, et al. (2009) Transport and release of chemicals from plastics to the environment and to wildlife. Philos Trans R Soc Lond B Biol Sci 364(1526):2027-2045.

12 GESAMP (2016) Sources, fate and effects of microplastics in the marine environment: part two of a global assessment. http://unesdoc. unesco.org/images/0024/002475/247517e.pdf.

13 Stufen in der Nahrungskette eines Ökosystems.

14 welt.de (2018) Mikroplastik gelangt über Mücken in Vögel und Fledermäuse. https://www.welt.de/newsticker/dpa_nt/infoline_nt/wis senschaft_nt/article181581212/Mikroplastik-gelangt-ueber-Mueckenin-Voegel-und-Fledermaeuse.html.

15 Triebskorn R, Braunbeck T, Grummt T, Hanslik L, Huppertsberg S, Jekel M, Knepper TP, Krais S, Müller YK, Pittroff M, Ruhl AS, Scmieg, H, Schür C, Strobel C, Wagner M, Zumbülte N, Köhler HR (2019) Relevance of nano- and microplastics for freshwater ecosystems: a critical review. Trends Analyt Chem 110:375-392.

16 Al-Jaibachi R, Cuthbert RN, Callaghan A (2018) Up and away: ontogenic transference as a pathway for aerial dispersal of microplastics. Biol Lett 14:20180479.
}

Rückgang von Vogelpopulationen damit in Verbindung gebracht. ${ }^{17}$ Auch ist noch nicht geklärt, ob Organismen oder Zellen, die beispielsweise mit Sandpulver bestreut würden, sich nicht genauso verhalten, als wenn sie Polymerpulver ausgesetzt werden.

\section{Mikroplastik in Lebensmitteln}

Plastikfasern und -fragmente finden wir aber nicht nur in Fischen, Mineralwasser, Meersalz, Honig oder Bier, sondern — mit geeigneten Messmethoden - überall. Vor allem dort, wo physikalische oder chemische Kräfte auf Plastik einwirken und Abrieb stattfindet. Schütteln Sie einmal Ihre kuschlige Mikrofaser-Bettwäsche im Sonnenlicht aus, so erkennen Sie zumindest den sichtbaren Anteil. Mit der Tasse Tee daneben, gekocht im Wasserkocher mit Kunststoffbehälter, ${ }^{18}$ können Sie ihren Tagesbedarf an Mikroplastik mit einem Schluck decken. Hausstaub besteht zu 33\% aus synthetischen Fasern, pro Tag rieseln zwischen 1600-11.000 Fasern auf einen Quadratmeter ihres Wohnraumes ${ }^{19}$ und ein entsprechender Anteil in ihre Teetasse. Catarino und Mitarbeiter ${ }^{20}$ haben ermittelt, wieviel Plastikfasern aus Muscheln in einer Mahlzeit im Vergleich zu den aus der Umgebungsluft auf den Teller rieselnden Fasern aufgenommen werden: 123-4620 MikroplastikFasern können je nach Konsum pro Jahr aus den Muscheln stammen, aber gleichzeitig fallen rund 13.700-76.400 Fasern aus der Umgebungsluft auf den Teller.

Und ist das denn nun ,schlimm“? Bisher sind trotz der all der oben aufgeführten Expositionen keine akuten, negativen Auswirkungen von Mikroplastik auf den Menschen bekannt. Unser Körper kann sehr gut mit oral aufgenommenen Partikeln oder Fasern umgehen, egal ob aus Sand oder Plastik, beides wird wieder ausgeschieden. Was wir allerdings noch nicht beurteilen können, sind die Langzeitauswirkungen eines permanenten oralen oder inhalativen Mikroplastik-Konsums. Oder was mit den bisher kaum erfassten sehr kleinen Mikroplastik-Partikeln

\footnotetext{
17 Der Tagesspiegel.de. Mikroplastik könnte über Mücken in Vögel und Fledermäuse gelangen (2018). https://www.tagesspiegel.de/ wissen/schadstoffe-in-der-nahrungskette-mikroplastik-koennte-uebermuecken-in-voegel-und-fledermaeuse-gelangen/23082554.html.

18 Wasserkocher: Gefahr durch Mikroplastik? https://www.ndr.de/ ratgeber/verbraucher/Wasserkocher-Gefahr-durch-Mikroplastik,mik roplastik272.html.

19 Dris R, Gasperi J, Mirande C, Mandin C, Guerrouache M, Langlois V, Tassin B (2017) A first overview of textile fibers, including microplastics, in indoor and outdoor environments. Environ Pollut 221:453-458.

${ }^{20}$ Catarino AI, Macchia V, Sanderson WG, Thompson RC, Henry TB (2018) Low levels of microplastics (MP) in wild mussels indicate that MP ingestion by humans is minimal compared to exposure via household fibres fallout during a meal. Environ Pollut 237:675-684.
} 
unter fünf Mikrometer oder gar mit Nanoplastik geschieht. Wegen diesen Unsicherheiten ist das Vorsorgeprinzip angebracht, und der Ruf nach geeigneten, standardisierten Messmethoden und Monitoringprogrammen berechtigt. Da die Partikel nicht weniger, sondern auf lange Sicht immer mehr werden, sollte das nicht nur für die humane Exposition, sondern auch für die Umwelt geschehen.

Wie wir Wissenschaftler die Ergebnisse derzeit kommunizieren und Medien Panik schüren ist problematisch.
Mediale Panikmache, blinder Aktionismus und Wetteifern um die nächsten, spektakulären Veröffentlichungen sind nicht angebracht. ${ }^{21}$ Vielmehr sollten eine zielgerichtete Forschung, eine realitätsnahe Risikobewertung, eine nachhaltige Kreislaufwirtschaft und eine Aufklärung des Verbrauchers erfolgen. Vor allem sollte eine Bewusstseinswerdung $\mathrm{zu}$ unserem verschwenderischen Konsum stattfinden.

„Ja, so einfach ist das wirklich nicht.”

${ }^{21}$ Backhaus T, Wagner M. 2018. Microplastics in the environment: Much ado about nothing? A debate. PeerJ Preprints 6:e26507v6 